\title{
Extraction and Analysis of Atomization Process from High-Fidelity Simulations
}

\author{
Brendan Christensen, Mark Owkes* \\ Department of Mechanical and Industrial Engineering, Montana State University, Bozeman, \\ MT, 59717-3800, USA \\ *Corresponding author email: mark.owkes@montana.edu
}

\begin{abstract}
Understanding the process of primary and secondary atomization in liquid jets is crucial in describing spray distribution and droplet geometry for industrial applications and is essential in the development of physics-based low-fidelity atomization models. Significant advances in numerical modelling and computational resources allows research groups to conduct detailed numerical simulations of these flows. However, the large size of the datasets produced by highfidelity simulations limit researchers' ability to analyze them. Consequently, the process of a coherent liquid core breaking into droplets has not been analyzed in simulation results even though a complete description of the jet dynamics exists. The present work applies a droplet physics extraction technique to high-fidelity simulations to track breakup events and data associated with the local flow. The data on the atomization process is stored in a Neo4j graphical database providing an easily accessible format. Results will provide a robust, quantitative description of the process of atomization and the details on the local flow field will be useful in the development of low-fidelity atomization models.
\end{abstract}

\section{Keywords}

jet breakup, physics extraction, atomization turbulence, direct numerical simulation

\section{Introduction}

Atomizing sprays have a wide range of applications in the automobile, aerospace, agricultural, and pharmaceutical industries. Consequently, the body of research on this topic is vast and spans multiple centuries. However, the mechanisms that drive instabilities in coherent liquid structures and ultimately breakup are still widely unresolved. This is, in part, due to limitations in experimental data collection from atomizing flows. Visual analysis of atomizing jets is difficult because of the scale and speed at which these systems develop. Additionally, the development of droplets creates an opaque cloud, blocking view of the liquid core, limiting understanding of primary instability and breakup mechanics. In the past decade, however, advances in computational resources have allowed research groups to develop direct numerical simulations (DNS) to better analyze atomization mechanisms. DNS provides significant advances in understanding of the underlying mechanisms that drive atomization. However, while these simulations produce a wealth of data, much of it has remained inaccessible due to the computational demand in parsing the massive datasets. This study's goal is to utilize the droplet ancestry extraction tool, first described in Rubel and Owkes [1], to access these previously unavailable statistics on droplet breakup to further understanding of the atomization process.

Among the first high resolution DNS studies on atomizing liquid jets were Desjardins and Pitsch [2] and Shinjo and Umemura [3]. These analyses were largely focused on describing breakup mechanisms over a range of bulk Weber and Reynolds numbers. They provided a wealth of information on mechanisms which were previously unclassified. Desjardins and Pitsch identified bubble formation through sweep-ejection events and ligament generation from bubble bursting and droplet collision. Shinjo and Umemura introduced a number of breakup 
modes as well, including short-wave breakup mode of ligaments and droplets from the jet tip and ligament formation from the jet core. Following these works, numerous other studies utilized increasingly high-resolution DNS to distinguish additional breakup processes and better describe already-identified mechanisms. Many of these works introduced vorticity information into their simulations in order to describe breakup in the context of vortex dynamics $[4,5,6,7,8]$. The above studies employed visual interpretations of the computed liquid-gas interface to characterize breakup mechanisms. The study described in Deshpande et al. [9] provides another interesting application of DNS in atomization research. The authors analyzed a simulated planar liquid jet with an emphasis on linear stability and the dominant wave mode in breakup. They found that the wavelength scales defined in standard linear stability theories were inconsistent with their simulations, provoking further research. The work described by Hasslberger et al. [10] paired a topological analysis with their DNS study to better classify flow behaviors throughout the simulation domain. The authors categorized all the small scale flow structures within an atomizing flow into four topologies. All of the aforementioned works provide a brief, but in no way comprehensive, overview of DNS atomization studies upon which the present study builds. Many atomization studies to date could be improved from quantitative descriptions of local breakup events and the ability to extract relevant data from discrete spatial and temporal locations within simulations.

Quantitative descriptions of breakup in literature are often limited by the measurement techniques available. They often rely on constrained temporal or spatial domains. Additionally, they often utilize global characteristics, such as the nozzle geometry or bulk velocities, despite local characteristics being more meaningful in predicting ligament and droplet breakup. Aerodynamic forces are understood to influence jet stability and contribute to breakup, however, this mechanism is not well understood because of difficulties collecting relevant quantities like local slip velocity and droplet characteristic lengths. A goal of this study is to extract values to use in analysis of local aerodynamic forces. A crucial parameter in quantifying breakup is the Weber number, defined as the ratio of inertial forces to surface tension forces. It is defined generally:

$$
\mathrm{We}_{\text {global }}=\frac{\rho U_{0}^{2} D_{0}}{\sigma}
$$

Most commonly, the global or bulk Weber number is measured, in which case $\rho$ is the density of the disruptive phase (gas in air-assisted atomization), $U_{0}$ is the bulk liquid velocity, $D_{0}$ is initial jet diameter (in some cases the radius), and $\sigma$ is the surface tension of the liquid $[2,4,7,8,11]$. Alternatively, the local Weber number, defined as

$$
\mathrm{We}_{\text {local }}=\frac{\rho U_{s}^{2} L}{\sigma},
$$

is only mentioned in a few studies, e.g. $[3,12,13,14]$. Where $U_{s}$ is the slip velocity defined as the difference in velocity between the liquid structure and the surrounding gas. $L$ is the characteristic length, which varies depending on the structure being analyzed. Because atomizing systems are dynamic and conditions vary throughout, the local Weber number is more consequential in describing liquid breakup. The prevalence of the global Weber number in literature can be attributed to the difficulty in measuring the slip velocity at discrete points throughout simulations or experiments. Salvador et al. [14] provide one of the very few simulation studies which displayed the local Weber number for specific droplets in a simulation. However, the authors were only able to calculate $\mathrm{We}_{\text {local }}$ for three unique droplets over three timesteps. The goal of the present study is to gather similar statistics for every droplet within a simulation at the precise timestep that it forms, which will provide useful insight into the conditions which lead to liquid breakup. 
A significant motivation for this work is to improve low-fidelity models. DNS relies on the complete resolution of the Navier-Stokes equations, which often requires high-performance computing and many days or weeks to perform the simulations. Many researchers and engineers do not have reasonable access to supercomputers or require faster simulation times, so they utilize low-fidelity atomization models to run simulations at reasonably low computational cost. These are often the combination of turbulence models, which model small scale turbulence and atomization models, which simplify interface deformation and transport. Commonly used turbulence models are large eddy simulations (LES) and the Reynolds averaged Navier-Stokes (RANS) equations. Many atomization models exist in literature. These include the Taylor analogy breakup (TAB) model [15], the Pilch-Erdman (PE) model [16], the Reitz-Diwakar (RD) model [17], the Eulerian-Lagrangian spray and atomization (ELSA) model [18], the bag type breakup (BTB) model [19], the multi-mode breakup (MMB) model [20], and the modified Taylor analogy breakup (MTAB) model [21].

All of these models try to predict how and when a liquid structure breaks apart. However, currently there is very little information on these events. The proposed tool provides a methodology to identify when a breakup event occurs. Additionally, the structure geometry and flow field can be assessed to quantify the details of the breakup conditions. This new information will advance our understanding of the atomization process and provide quantitative information to inform and improve reduced order atomization models.

\section{Computational Fluid Dynamics Solver}

The droplet extraction tool was implemented into the NGA computational platform [22, 23, 24, 25]. NGA solves mass and momentum conservation equations on a staggered Cartesian mesh, in which scalar values, such as pressure, are stored at cell centers and velocities at the cell faces. Time is discretized utilizing an iterative Crank-Nicolson formulation with a semi-implicit correction on each sub-iteration [26]. The liquid interface is identified using an un-split semiLagrangian volume-of-fluid method (VOF) that conserves both mass and momentum [25]. The interface is reconstructed using a piecewise linear interface reconstruction (PLIC) [27] with interface normal vectors computed with efficient least-squares VOF interface reconstruction algorithm (ELVIRA) [28]. The surface tension force is computed with the Adjustable Curvature Evaluation Scale (ACES) method [29]. NGA is fully parallelized with message passing interface (MPI) and scales well to tens-of-thousands of cores [2].

While NGA is used in this work, the proposed extraction tool could be applied to other highfidelity solvers. The tool requires a scalar that moves with liquid structures and a band growth algorithm to assign a unique number to each structure. With these two inputs, the tool provides a methodology to identify split and merge events and a procedure to export and analyze the data.

\section{Droplet Extraction Tool}

The droplet genealogy extraction tool, which is described in detail in Rubel and Owkes [1], identifies splitting and merging events within an atomization simulation. Relevant data from each event is extracted and written to a CSV file, then stored in a neo4j graphical database. Splits and merges of liquid structures are identified using two unique integer identification numbers assigned to each droplet. First, the structure identification number $(\mathcal{S})$ is unique to every independent liquid structure within the simulation. $\mathcal{S}$ values are assigned at each timestep using a band-growth algorithm [30]. $\mathcal{S}$ is not persistent through time and the $\mathcal{S}$ value may vary between timesteps. Liquid identification numbers $(\mathcal{L})$ are transported with the liquid it is associated with until a split or merge event is identified, and then the $\mathcal{L}$ value is changed. A split is identified when the same $\mathcal{L}$ exists in more than one structure. In other words, a different $\mathcal{S}$ is associated 
with the same $\mathcal{L}$. A merge is identified when multiple $\mathcal{L}$ exist within the same structure (one $\mathcal{S}$ ). See Figure 1 for an illustration of this process.

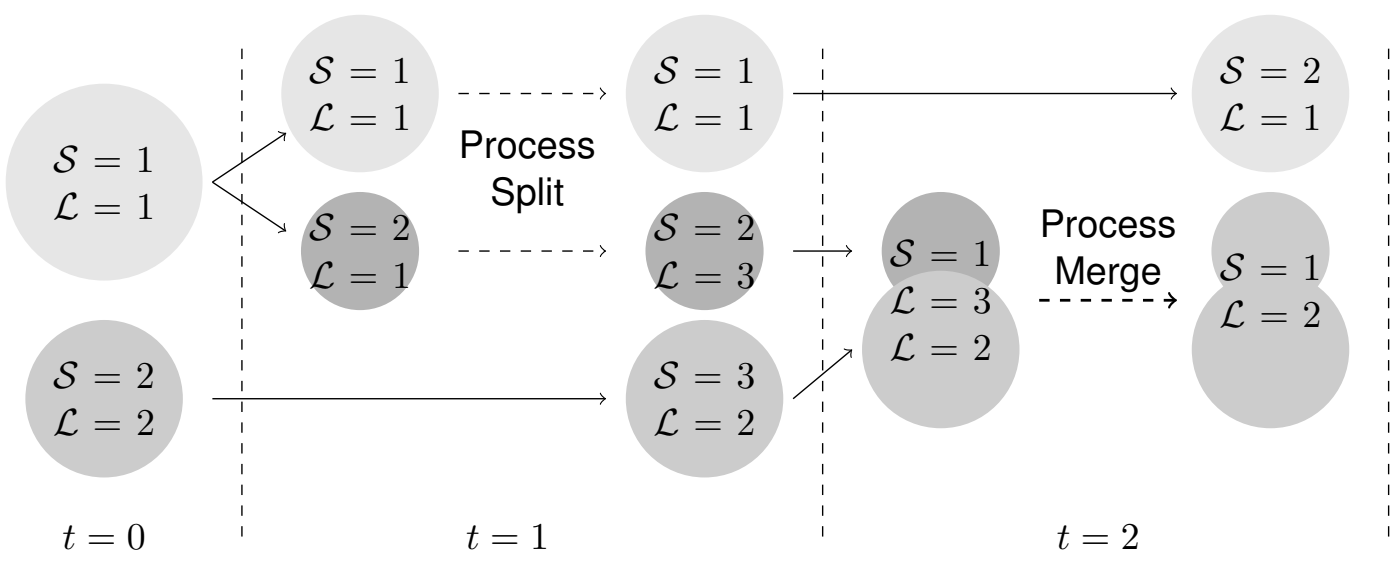

Figure 1. An example of two droplets undergoing a split and a merge. Note that the $\mathcal{S}$ value can vary between timesteps, but the $\mathcal{L}$ is persistent until a split or merge causes the value to change.

An issue from the original work, described in Rubel and Owkes [1] was the occurrence of numerous fictitious merge and split events. These events occurred when a droplet split, meaning there was one full cell between it and the parent droplet. Then, as the parent droplet continued to advance, the droplets remerged when they were in adjacent cells. To avoid an accumulation of non-physical results, the authors opted to run the split identification portion of the code every 10 timesteps. The present work changed the parameters that defined merge events, so that merges only occur when two structures exist within the same cell rather than in adjacent cells. Splits still occur when there is a full cell between the two structures. Using a larger separation distance for splits compared to merges allows for the split and merge identification tool to run on every timestep, bolstering its accuracy and usefulness.

\section{Simulation Setup}

This work analyzed a three-dimensional diesel jet to demonstrate the droplet extraction tool's usefulness. The test case is identical to the simulation analyzed in Rubel and Owkes [1]. Shape characterization and local flow field values are extracted and the code is updated to sample at every timestep, so investigating the same test case will demonstrate the improvements to this method. The simulation was run on roughly 17 million computational cells $\left(N_{x}=1024\right.$, $N_{y}=128, N_{z}=128$ ). With this grid resolution, and the jet statistics in Table 1, this simulation was able to run in less than a week on 160 processors on the Hyalite High-Performance Computing System. The above resolution is not sufficient to fully resolve the smallest scales within a physical atomizing system. These results are preliminary, and future work will further address grid resolution and simulation accuracy.

\section{Results and discussion}

A diesel jet, as described above, was simulated to demonstrate the droplet extraction tool's utility. Further simulations at varying conditions and higher resolution will be run in order to draw concrete analyses, but preliminary results show promise for the method's usefulness. This simulation was run for a relatively short period of time, producing roughly 1100 droplets. Due to the relatively coarse grid resolution, droplets less than 1/100th of the jet diameter were discarded, because they could not be confidently resolved. This resulted in 949 droplet breakup events that were analyzed.

Figures 3 and 4 demonstrate how spatial distributions of breakup events can be displayed 


\begin{tabular}{|c|c|c|}
\hline Number & Definition & Value \\
\hline Bulk Reynolds number & $\rho_{l} U_{\text {jet }} D_{\text {jet }} / \mu_{l}$ & 25,000 \\
Bulk Weber number & $\rho_{l} U_{\text {jet }}^{2} D_{\text {jet }} / \sigma_{l}$ & 10,000 \\
Density ratio & $\rho_{l} / \rho_{g}$ & 40 \\
Viscosity ratio & $\mu_{l} / \mu_{g}$ & 1.67 \\
Domain length & $L_{x} / D_{\text {jet }}$ & 60 \\
Domain widths & $L_{y, z} / D_{\text {jet }}$ & 7.5 \\
Cells across diameter & $D_{\text {jet }} / \Delta \mathrm{x}$ & 17.06 \\
CFL number & $|u|_{\text {max }} \Delta t / \Delta x$ & 0.4 \\
\hline
\end{tabular}

Table 1. Non-dimensional parameters used in the diesel jet simulation.

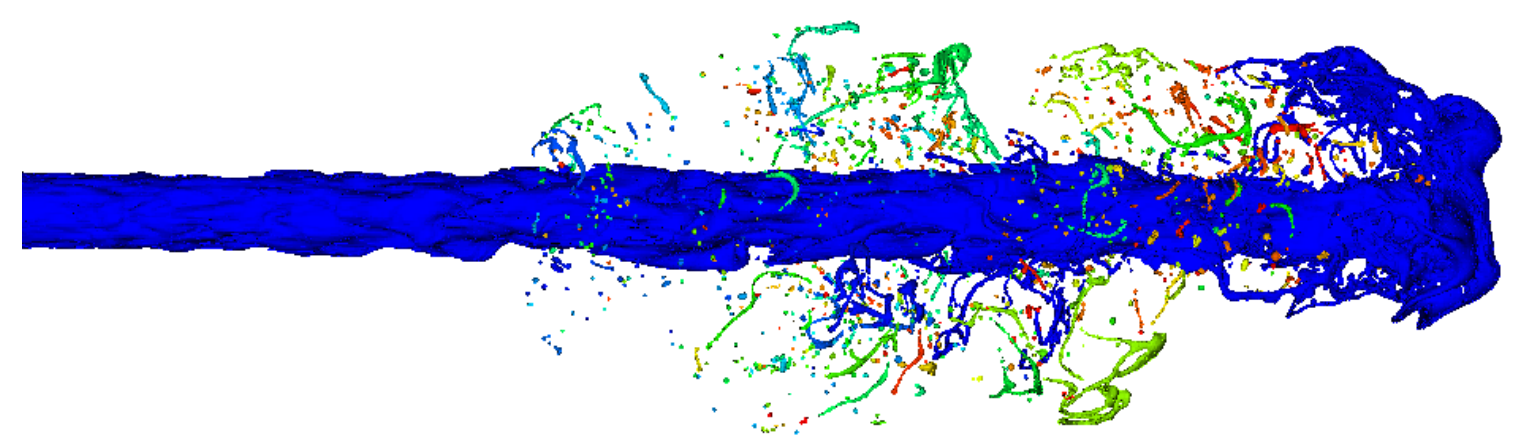

Figure 2. Simulated jet used described in table 1 simulated to $1 \mu \mathrm{s}$. The colors represent $\mathcal{L}$ at this final timestep.

using data extracted from this method. The former shows the locations of all the primary and secondary breakup events within the simulation relative to the spray core (at the center of the plot). Figure 4 shows the non-dimensional axial distance from the nozzle and the local Weber number of primary droplets calculated using the current timestep slip velocity and the characteristic length equal to $D_{\text {jet }}$. The figure is consistent with the findings of Shinjo and Umemura [3], in which they showed that breakup occurs when the inertial forces balance with the surface tension force, i.e. when $\mathrm{We}_{\mathrm{g}} \sim \mathcal{O}(1)$. While there is some spread in this data, the values are centered about $\mathcal{O}(1)$. Future work will incorporate the local Weber numbers for secondary breakup events by sampling from data at the previous timestep, which will allow for identification of the local flow field and characteristic lengths of droplets immediately preceding breakup.

Of significant interest in atomization modelling is droplet shape and size distribution. These are values which can be easily extracted with the use of the described tool. A simple shape parameter was implemented using the three principle axes of each droplet. The smallest axis (b) was divided by the major axis $(a)$, which results in values close to one for spherical droplets and close to zero for ligaments. Figure 5 shows an interesting distribution for droplets which just underwent breakup. Both primary and secondary droplets show a distribution peak around $b / a=0.2$. With a larger dataset, probability density functions can be built at progressive timesteps to show how droplet size and shape vary as a function of time or location within an atomizing simulation. This will be completed in future work with higher resolution simulations and varying jet conditions. 


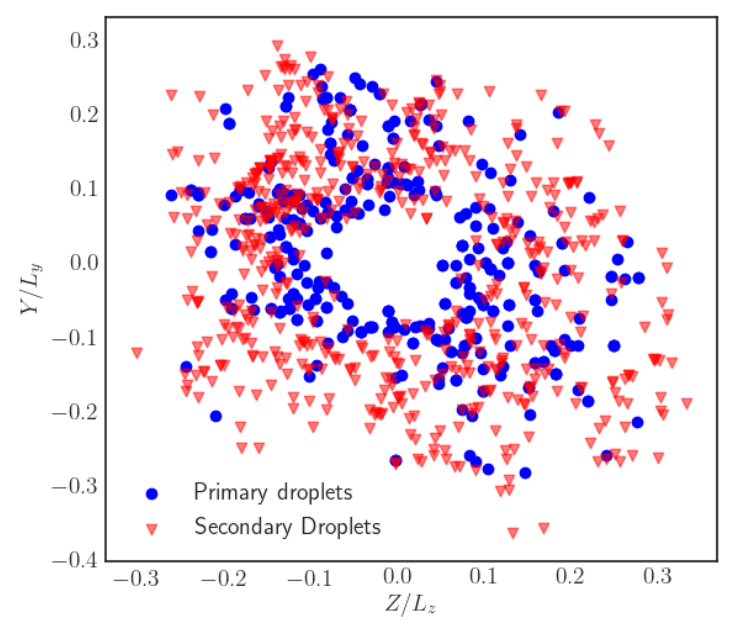

Figure 3. Spatial distribution of all primary and secondary breakup events through $1 \mu \mathrm{s}$ in the Y-Z plane, i.e. perpendicular to the jet axis.

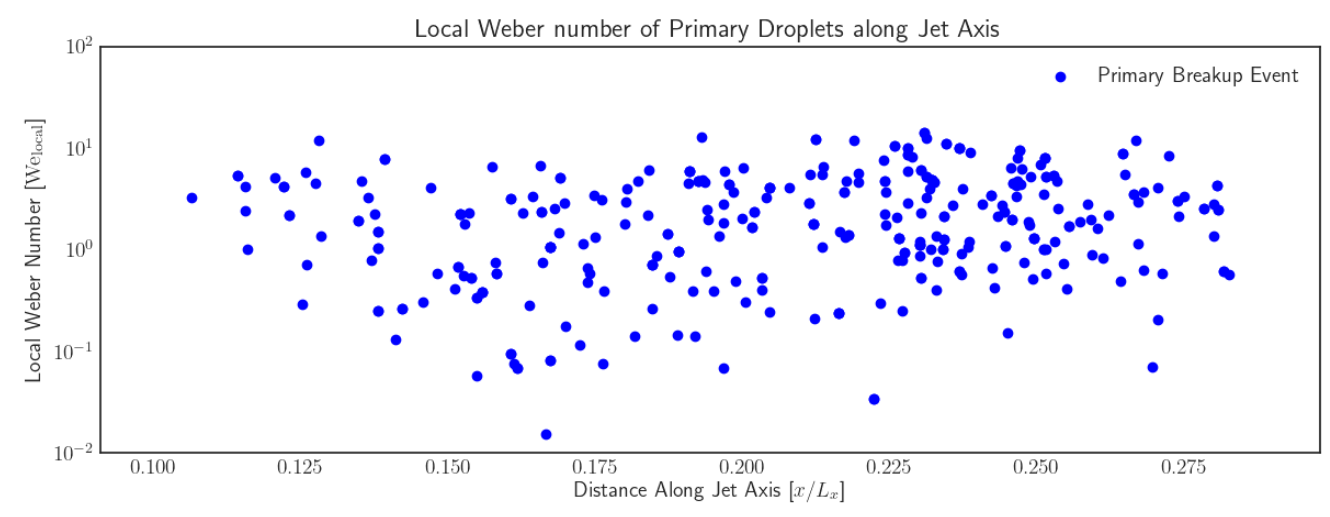

Figure 4. Local Weber number and distribution of primary breakup events along the jet axis.
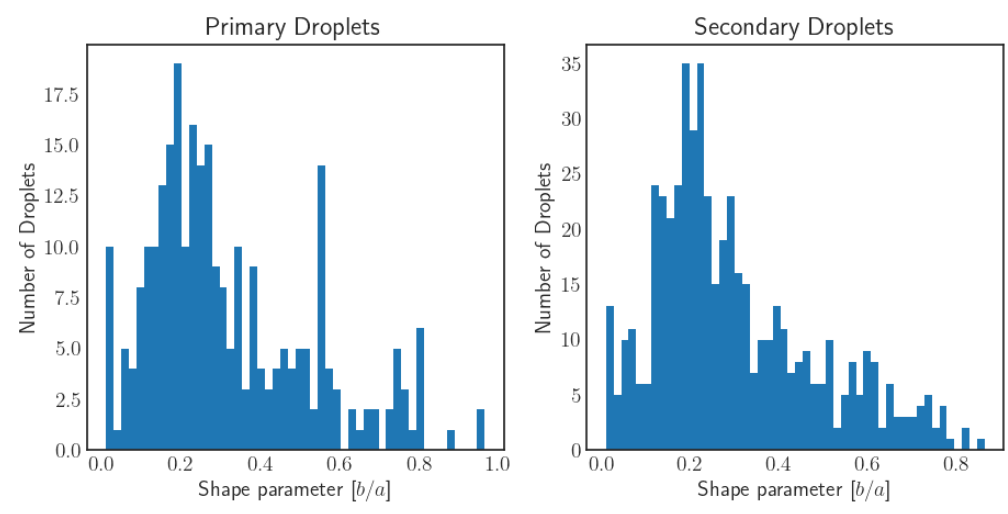

Figure 5. Distribution of the shape parameter $b / a$ for primary and secondary droplets. 


\section{Conclusions}

The tool described in this work allows for the extraction of local characteristics important to the atomization process, which were previously unobtainable in DNS. The ability to relate spatial and temporal positions of breakups, local flow field attributes, and shape characteristics of atomizing droplets provide a plethora of new information for atomization model developers. Future work will focus on simulating physically resolved jets and extracting comprehensive information about every breakup event, elucidating the droplet formation process.

\section{Acknowledgements}

This material is based upon work supported by the National Science Foundation under Grant No. 1749779.

Computational efforts were performed on the Hyalite High Performance Computing System, operated and supported by University Information Technology Research Cyberinfrastructure at Montana State University.

\section{References}

[1] Rubel, Clark and Owkes, Mark. "EXTRACTION OF DROPLET GENEALOGIES FROM HIGH-FIDELITY ATOMIZATION SIMULATIONS." Atomization and Sprays Vol. 29 (2019): pp. 709-739. .

[2] Desjardins, O and Pitsch, H. "DETAILED NUMERICAL INVESTIGATION OF TURBULENT ATOMIZATION OF LIQUID JETS." Atomization and Sprays Vol. 20 (2010): pp. 311-336.

[3] Shinjo, J. and Umemura, A. "Simulation of liquid jet primary breakup: Dynamics of ligament and droplet formation." International Journal of Multiphase Flow Vol. 36 (2010): pp. 513532. .

[4] Jarrahbashi, D. and Sirignano, W. A. "Invited Article: Vorticity dynamics for transient highpressure liquid injection." Physics of Fluids Vol. 26.

[5] Watanabe, Tomoaki, Sakai, Yasuhiko, Nagata, Kouji, Ito, Yasumasa and Hayase, Toshiyuki. "Vortex stretching and compression near the turbulent/non-turbulent interface in a planar jet." Journal of Fluid Mechanics Vol. 758 (2014): pp. 754-785. .

[6] Jarrahbashi, D., Sirignano, W. A., Popov, P. P. and Hussain, F. "Early spray development at high gas density: Hole, ligament and bridge formations." Journal of Fluid Mechanics Vol. 792 (2016): pp. 186-231.

[7] Zandian, A., Sirignano, W. A. and Hussain, F. "Planar liquid jet: Early deformation and atomization cascades." Physics of Fluids Vol. 29. .

[8] Zandian, A., Sirignano, W. A. and Hussain, F. "Understanding liquid-jet atomization cascades via vortex dynamics." Journal of Fluid Mechanics Vol. 843 (2018): pp. 293-354.

[9] Deshpande, Suraj S., Gurjar, Soumil R. and Trujillo, Mario F. "A computational study of an atomizing liquid sheet." Physics of Fluids Vol. 27 (2015): p. 082108. .

[10] Hasslberger, Josef, Ketterl, Sebastian, Klein, Markus and Chakraborty, Nilanjan. "Flow topologies in primary atomization of liquid jets: A direct numerical simulation analysis." Journal of Fluid Mechanics Vol. 859 (2019): pp. 819-838. .

[11] Sallam, K A, Dai, Z and Faeth, G M. "Liquid breakup at the surface of turbulent round liquid jets in still gases." International Journal of Multiphase Flow Vol. 28 (2002): pp. 427-449.

[12] Umemura, Akira and Osaka, Jun. "Self-destabilizing loop observed in a jetting-to-dripping transition." Journal of Fluid Mechanics Vol. 752 (2014): pp. 184-218.

[13] Pham, P. X., Kourmatzis, A. and Masri, A. R. "Local characteristics of fragments in atomizing sprays." Experimental Thermal and Fluid Science Vol. 95 (2018): pp. 44-51. .

[14] Salvador, F. J., Ruiz, S., Crialesi-Esposito, Marco and Blanquer, Ignacio. "Analysis on 
the effects of turbulent inflow conditions on spray primary atomization in the near-field by direct numerical simulation." International Journal of Multiphase Flow Vol. 102 (2018): pp. 49-63. .

[15] O'Rourke, Peter J. and Amsden, Anthony A. "The Tab Method for Numerical Calculation of Spray Droplet Breakup." 1987.

[16] Pilch, $M$ and Erdman, $C$ A. "USE OF BREAKUP TIME DATA AND VELOCITY HISTORY DATA TO PREDICT THE MAXIMUM SIZE OF STABLE FRAGMENTS FOR ACCELERATION-INDUCED BREAKUP OF A LIQUID DROP." Int. J. Multiphase Flow Vol. 13 (1987): pp. 741-757.

[17] Reitz, Rolf D. and Diwakar, R. "Structure of High-Pressure Fuel Sprays." 1987. .

[18] Wang, Yue, Lee, Won Geun, Reitz, Rolf D. and Diwakar, Ramachandra. "Numerical Simulation of Diesel Sprays Using an Eulerian-Lagrangian Spray and Atomization (ELSA) Model Coupled with Nozzle Flow." 2011. .

[19] Wang, C., Chang, S., Wu, H. and Xu, J. "Modeling of drop breakup in the bag breakup regime." Applied Physics Letters Vol. 104. .

[20] Wang, C, Chang, S, Wu, H, Ding, L and Thompson, J M. "THEORETICAL MODELING OF SPRAY DROP DEFORMATION AND BREAKUP IN THE MULTIMODE BREAKUP REGIME." Atomization and Sprays Vol. 25 (2015): pp. 857-869.

[21] Sula, C., Grosshans, H. and Papalexandris, M. V. "Assessment of Droplet Breakup Models for Spray Flow Simulations.” Flow, Turbulence and Combustion Vol. 105 (2020): pp. 889914. .

[22] Desjardins, Olivier, Blanquart, Guillaume, Balarac, Guillaume and Pitsch, Heinz. "High order conservative finite difference scheme for variable density low Mach number turbulent flows." Journal of Computational Physics Vol. 227 (2008): pp. 7125-7159. .

[23] Desjardins, Olivier, Moureau, Vincent and Pitsch, Heinz. "An accurate conservative level set/ghost fluid method for simulating turbulent atomization." Journal of Computational Physics Vol. 227 (2008): pp. 8395-8416. .

[24] Owkes, Mark and Desjardins, Olivier. "A computational framework for conservative, threedimensional, unsplit, geometric transport with application to the volume-of-fluid (VOF) method." Journal of Computational Physics Vol. 270 (2014): pp. 587-612. .

[25] Owkes, Mark and Desjardins, Olivier. "A mass and momentum conserving unsplit semiLagrangian framework for simulating multiphase flows." Journal of Computational Physics Vol. 332 (2017): pp. 21-46. .

[26] Choi, Haecheon and Moin, Parviz. "Effects of the Computational Time Step on Numerical Solutions of Turbulent Flow." Journal of Computational Physics Vol. 113. .

[27] Youngs, David L. Time-Dependent Multi-material Flow with Large Fluid Distortion. Academic Press (1982): pp. 273-285.

[28] Pilliod, James Edward and Puckett, Elbridge Gerry. "Second-order accurate volume-offluid algorithms for tracking material interfaces." Journal of Computational Physics Vol. 199 (2004): pp. 465-502.

[29] Owkes, Mark, Cauble, Eric, Senecal, Jacob and Currie, Robert A. "Importance of curvature evaluation scale for predictive simulations of dynamic gas-liquid interfaces." Journal of Computational Physics Vol. 365 (2018): pp. 37-55. .

[30] Herrmann, M. "A parallel Eulerian interface tracking/Lagrangian point particle multi-scale coupling procedure." Journal of Computational Physics Vol. 229 (2010): pp. 745-759. . 